eigentlich zusammen eine dritte den Malveen und Ureneen gleichwerthige Gruppe von Gewächsen. Aber in Anbetracht ihrer grossen Zahl, auch in Anbetracht des Umstandes, dass viele Botaniker auf das Verwachsenbleiben oder die Sonderung der einzelnen Karpelle bei der Fruchtreife ein grösseres Gewicht legen, als ich es vermag, glaube ich eher Beifall für meine Anordnung der Malvaceen zu erhalten, wenn ich für die übrigen Malvaceen noch zwei Subordines "der Sideae und Hibisceae" vorzuschlagen mir erlaube.

Was meine Tribus anbelangt, so mögen diese für sich selbst reden. Sie sind einfach charakterisirt und weiss ich vorerst keine besseren aufzustellen. Das in Obigem von mir empfohlene Malvaceensystem mag das erste sein, das nirgends der Hülle gedenkt, während bisher und noch bei Gray auf dies Merkmal ein über Gebühr grosses Gewicht gelegt wurde. Man wird auch sehen, dass wir dieses Merkmales bei einer Eintheilung der Malvaceen gar nicht . bedürfen, da wir wichtigere Unterscheidungsmerkmale bei ihnen dazu finden. Ich für meinen Theil kann der An- oder Abwesenheit und Beschaffenheit der Hülle nur in sehr wenig Fallen nur Gattungswerth zugestehen (wie I'Heritier stirp. nov. p. 133), finde sie aber sehr bequem zur Auffindung der Arten in einer Gattung. 0 berramstadt im Februar 1862.

\title{
Ein Ausflug auf den Thebner Kobel.
}

Von Karl Brancsik.

Es war am 30. Mai 1858, als ich von Pressburg aus einen Ausflug nach dem Thèbner Kobel unternahm *).

Hat man die letzten Häuser Pressburgs am linken Ufer der Donau im Rücken, so entfernt sich auch der Weg immer mehr vom Ufer und führt zwischen Weingärten und Fruchtfeldern bis zum Orte Karlsdorf, von wo er sich wieder dem Stromufer nähert. Die Weingärten verschwinden dann, links wogt die Donau und rechts erhebt sich eine Hügelkette, die öfters von Querthälern durchschnitten wird. Sanft erhebt sich nun der Weg, indem er einen Hohlweg bildet, senkt sich zu Ende des letzleren um sich bald wieder zu erheben, bis er eine Höhe erreicht, von welcher man die letzte Aussicht über Pressburg und die Ebene, welche sich gegen Kittsee und den Neusiedlersee hinzieht, geniessen kann. Auf diesem Weg sammelte ich: Vicia pannonica, Lithospermum purpureo coeruleum,

*) Im 3. Jahrgange unserer Zeitschrift (Oesterr. botan. Wochenblatt, 1853, Seite 410) wird von Adolf $\mathrm{D}$ ietl ein Ausflug auf den Thebner Kobel beschrieben, welchen der bemerkte Botaniker am 22. August 1853 ausgeführt hat. Cytisus argenteus L., den Dietl bei jener Gelegenheit gefunden haben wollte, hat sich später als Cytisus austriacus L. erwiesen.

Die Redact. 
Diplotaxis muralis, Farsetia incana, Thlaspi perfoliatum, Lepidium Draba, Fumuria officinalis, Sisymbrium Loeselii, S. Sophia, Hotosteum umbellatum, Stellaria Holostea, Ornithogalum umbellatum, Carex muricata u. a.

Mittlerweile gelangt man zu einem Gehöfte, das einsam neben dem Wege steht und zu einem Wäldchen, in welchem ich Chaerophyllum temulum und Ch. aromaticum fand. Hat dieses Wäldchen mir bisher theilweise Schatten gespendet, so musste ich nun eine Strecke auf dem sich immer mehr erhebenden Terrain unter den sengenden Strahlen der Sonne wandeln. Doch bald gewinnt der Weg an Annehmlichkeit, zu seinen beiden Seiten neigen Bäume ihre Aeste gegen einander, so dass sie sich verschlingend, ein undurchdringliches Blätterdach bilden. Auf einer Gartenwiese nebenan kommt Orchis coriophora vor, ich fand sie nicht, dagegen Saxifraga tridactylites, $S$. bulbifer ${ }^{2}$ und Trifolium alpestre. In dem nahestehenden Garten neben Pulmonaria officinalis dufteten Convallaria majalis und Asperula odorata. $\mathrm{Zu}$ beiden Seiten des Weges stehen Viburnum Lantana, Prunus Mahaleb, Staphylea pinnata, Acer campestre, Evonymus europaeus, Rosa canina und Crataegus monogyna, hie und da von Clematis Vitalba durchrankt. Weiter schreitend erreicht man ein Jägerhaus, vor welchem zwei mächtige Eichen (Quercus pubescens) stehen und in dessen Nähe sich der erste Steinbruch zeigt, deren es in der Gegend noch mehrere gibt. Gegenüber dem Jägerhause windet sich ein schmales Kreuzthal, das von einem Bächlein durchrieselt wird. Neben einer Quelle fielen mir eiñige blüthenlose Pflanzen der hier seltenen Salvia glutinosa auf, welche ich auch später nie blühend traf. Ihr scheint die Gegend nicht günstig zu sein, während sie weiter gegen Trenchin und in den Karpaten sehr häufig vorkommt, Auf den Abhängen des Thales fand ich zwischen Bäumen Viola mirabilis, bereits zum zweiten Male blühend, dann Arabis hirsuta.

Das Thal verlassend wandte ich mich wieder dem Jägerhause zu und schritt, um den Weg abzukürzen durch einige Gärten, da erhob sich aus dem Grase eine Schlange, mit einem Hiebe meines Stockes tödtete ich sie, es war ein Coluber Natrix. Auf den Gartenwiesen fand ich Campanula sibirica, Orchis ustulata, Polygala vulgaris und Tragopogon undulatifolius. Ich eilte weiter bis zu den felsigen Abhängen, die sich gegen die Donau neigen. Hier ändert sich merklich die Vegetation. In grosser Menge erscheint Xeranthemum annuum, dann Bromus squarrosus, Hieracium praealtum Vill. Allium sphaerocephalum, Alyssum calycinum, Reseda Iutea, Delphinium Consolida, Papaver dubium und Arabis arenosa. Näher gegen Theben bemerkte ich neben dem Wege Centaurea axillaris (nicht blühend), Jasione montana, Dianthus prolifer und Echinops sphaerocephalus. Auf den Felsen sammelte ich Teucrium Chamaedris, Alyssum montanum, Sedum album; Inula Oculus Christi stand noch nicht in Blüthe; dagegen war Iris pumila bereits 
verblüht. Vor Theben fand ich noch Glaueium corniculatum und Erysimum austriacum.

Theben, einst eine mächtige Veste, jetzt ein Trümmerhaufen, bildet mit dem gegenüber liegenden Hainburg die "Porta Hungariae“, durch die sich die Donau vereinigt mit der March wälzt. Um auf den Kobel zu gehen, muss man die Stadt durchschreiten, bei der IKirche gelangt man zum sandigen Wege auf denselben, die Schlucht, durch welche er führt, ist aus Sandstein gebildet, während der $1000^{\prime}$ hohe Kobel selbst aus Granit besteht. Ausserhalb der Schlucht fand ich auf Sandboden Prunus Chamaecerasus, Silene Otites, Hieracium echioides (nicht blühend), Globularia vulgaris, Statice elongata, Cytisus capitatus und C. austriacus (nicht blühend). Einige nicht ferne Vertiefungen beherbergten Anemone sylvestris, Helianthemum vulgare, Adonis aestivalis, Polygala major, Alsine Jacquenii, Al. tenuifolia, Linum tenuifolium (nicht blühend), Anthericun ramosum, Anthyllis Vulneraria, Campanula glomerata; dann Anemone pratensis und A. Pulsatilla aber bereits mit Früchten.

Ein schlechter Fahrweg führt auf die Erhöhung des Kobels, die Weingärlen sind verschwunden und nur starre Felsen, bedeckt mit Alyssum montanum, Poa alpina und Teucrium montanum, blicken aus dem Berge hervor, zwischen diesen krümmt sich Rhamnus saxatilis und Cotoneaster vulgaris, im Gesträuch wächst Anemone sylvestris und den Boden überziehen Cytisus biflorus, Genista pilosa und Dirycnium suffruticosum. Gemein ist daselbst Adonis aestivalis, Arabis .Turrita und Scorzonera austriaca. Weiter oben bedeckt den Berg Gesträuch von Cornus mas, C. sanguinea, Acer campestre, Evonymus verrucosus, E. europaeus dazwischen auf feuchlen Stellen kommt Orobus albus vor und weiter gegen Neudorf hin das schöne Smyrrium perfoliatum Mill. An den Abhängen des Kobels, gegen die March $\mathrm{zu}$, fand ich Verbascum phoeniceum mitunter mit weissen Blüthen. Auf den Felsen stehen Iris pumila, Jurinea mollis, Clematis integrifolia, Scorzonera austriaca, Vinca herbacea W. K., und Viola hirta, auf Grasplätzen Carex humilis, Sipa pennata und Medicago minima. Hier befindet sich auch der Standort der Euphorbia Gerardiana $\beta$. Surii Holuby, Hesperis tristis und Orchis pallens, die hier ebenfalls vorkommen, habe ich nicht gefunden. Schliesslich bemerke ich noch, dass der Kobel eine Flora besitzt, die sich von der aller umliegenden Berge auffallend unterscheidet. Eine Aehnlichkeit mit derselben finde ich hier um Oedenburg, auf den Bergen, die am Neusiedlersee liegen und worüber ich mir später einige Bemerkungen zu machen erlauben werde.

Oedenburg, im Februar 1862. 